\title{
The Novel Hub Genes of Klinefelter Syndrome Affect Sperm Cell Differentiation: Analysis Based on RNA-Seq and scRNA-Seq Data
}

\section{Hai-Hong He}

Shenzhen hospital, Southern Medical University

Fan Yu

Shenzhen hospital, Southern Medical University

Ting-Ting Huang

Shenzhen hospital, Southern Medical University

Ke-Yan Chen

Shenzhen hospital, Southern Medical University

\section{Shi-Xing Guo}

Shenzhen hospital, Southern Medical University

\section{Li-Jun Zhang}

Shenzhen hospital, Southern Medical University

\section{Xi Tang}

Shenzhen hospital, Southern Medical University

\section{Xin-Hua Yuan}

Shenzhen hospital, Southern Medical University

Yi-Wen Zhou (D1147579905@qq.com )

Shenzhen hospital, Southern Medical University

Jiao Liu

Shenzhen hospital, Southern Medical University

\section{Research Article}

Keywords: Single cell sequencing, Klinefelter syndrome, hub genes, sperm cell, KIF2C

Posted Date: January 24th, 2022

DOI: https://doi.org/10.21203/rs.3.rs-1198365/v1

License: (a) (i) This work is licensed under a Creative Commons Attribution 4.0 International License.

Read Full License 


\section{Abstract \\ Objective}

In this study, the Hub genes that are closely associated with spermatogenesis in klinefelter syndrome (KS) patients were identified, mainly through transcriptomic and single-cell RNA sequencing (scRNAseq)data.

\section{Methods}

Transcriptome data were obtained from 3 adult 4 fetal KS patients and 8 normal individuals. ScRNA-seq data were obtained from 1 adult KS patient and 4 normal individuals. The differentially expressed genes (DEGs) were analysed using limma's method. The testicular tissue-specific protein-protein interaction (PPI) networks were analysed through the NetworkAnalyst website. The CytoHubba plugin was used to draw Veen Diagram. Integration of scRNA-seq data using the Seurat package. Developmental trajectories of sperm cells and hub genes were constructed by using the monocle3.

\section{Results}

The results of the GSE103905 and GSE103613 transcriptome datasets show a total of 700 DEGs. Gene Ontology (GO) analysis focused on memory, methylation, ATPase activity and mitochondrion. Kyoto Encyclopedia of Genes and Genomes (KEGG) focused on metabolic pathways, huntington's disease and nicotinamide metabolism. Four Hub genes (KIF2C, MRPS2, RPS15, TSFM) that have not been reported to be associated with KS were identified. Single-cell sequencing data showed that only KIF2C and RPS15 were expressed in sperm cells. hub genes' Pseudotime analysis showed a decreasing trend in RPS15 expression and an increasing trend in KIF2C expression throughout sperm development.

\section{Conclusion}

In this study, two RPS15 and KIF2C were identified that may be closely associated with spermatogenesis in KS patients, but there may be differences in the mechanisms by which they affect sperm development.

\section{Background}

Klinefelter syndrome, the most common type of sex chromosome abnormality in men, is recognised worldwide as the leading genetic factor causing male infertility[1]. KS has a prevalence of about $0.1-0.2 \%$ in neonatal boys, accounts for about $3 \%$ of male infertility patients and is responsible for about $10-12 \%$ of non-obstructive azoospermia patients[2]. This percentage is even higher in China: $16.5 \%$ in the south of the country, $14.1 \%$ in the north and $11.2 \%$ in Hong Kong. Data from our previous study in the region 
showed that KS accounted for $12.62 \%$ of NOA and was also the primary genetic factor contributing to male infertility[3].

The clinical phenotype of KS is complex and varied, typically characterised by sparse body hair, feminised gynecomastia, short penis and small, hard testicles[4]. 80-90\% of KS patients fall into the $47, X X Y$ karyotype, with chimerism and X chromosome structural abnormalities accounting for the remaining $10 \%[5]$. From infancy to adulthood, and especially after puberty, the number of germ cells in KS patients is progressively reduced, with only a very small number or complete absence

of germ cells in adulthood, eventually leading to male infertility[6]. With advances in assisted reproduction techniques such as testicular sperm extraction (TESE) and intracytoplasmic sperm injection (ICSI) combined, it is possible for $30-66 \%$ of KS patients to have biological offspring[7-9], but more than half of KS patients still do not have access to genetic offspring. Therefore, understanding the key factors that influence the process of sperm development will bring light to KS patients who are unable to reproduce offspring.

The extra X chromosome in KS patients may be of paternal or maternal origin, with a slightly higher probability of being maternal than paternal (55\% vs. $45 \%)[10,11]$.In patients with KS, current studies in the literature have found that additional genes on the X chromosome exhibit escape inactivation, allowing for abnormal gene expression in male individuals, resulting in the production of altered mRNA and gene products that may correlate with the severity of the KS phenotype.The pathogenesis of spermatogenesis disorders due to abnormal gene expression in KS patients is poorly understood and still needs further elucidation[12, 13].

In the current study, we utilised gene expression profiling microarray transcriptomic data and scRNA-seq data from the GEO database. DEGs were obtained by analysing gene expression transcriptomic data from KS and normal human testicular tissue. We then performed GO and KEGG enrichment

analyses and further identified hub genes by PPI network analysis. We identified hub genes expressed in sperm cells by integrating scRNA-seq data from KS and normal human testicular cells. The role of hub genes in sperm development was revealed by proposed temporal sequence analysis.

\section{Materials And Methods}

\section{Research data}

GEO (http://www.ncbi.nlm.nih.gov/geo) is one of the free and data-rich public genomic databases, containing various types of gene microarray data, microarray and single cell sequencing data, etc. The gene expression transcriptome data for this study were derived from the GSE103905 and GSE103613, with the GSE103905 data derived from 3 adult KS samples and 3 adult, and the GSE103613 data derived from 4 fetal KS samples and 5 adult controls. The scRNA-seq data were derived from the GSE130151 and 
GSE124263, with the GSE130151 dataset derived from testicular tissue from 1 adult KS and the GSE124263 dataset consisting mainly of 4 normal human testicular cells.

\section{Screening for EEGs}

DEGs were identified by the limma package (version 3.46.0) of R software (version 4.02) with screening thresholds set at $\mid \log 2 \mathrm{FCl}>1.5$ and $\mathrm{P}<0.05$.

\section{GO and KEGG enrichment pathway analyses}

GO and KEGG enrichment pathway analyses were performed on DEGs using the DAVID (version 6.8) online website (https://david.ncifcrf.gov/). GO functional analyses were performed mainly by BP, MF and $\mathrm{CC}$ and using the ggplot2 package (version 3.3.5) for bubble map visualisation analysis.

\section{PPI Network Construction and Identification of hub genes}

The NetworkAnalyst website (https://www.networkanalyst.ca/)[14] was used to construct a testis tissuespecific PPI network of DEGs. The PPI networks were imported into Cytoscape software (version 3.6.1), and the CytoHubba plugin was used to Degree, Density of Maximum Neighborhood Component

(DMNC), Maximal Clique Centrality (MCC) and Maximum neighborhood component (MNC) four methods to draw Veen Diagram and finally identify hub genes.

\section{Integration of single-cell sequencing data, cell clustering and annotation, Hub genes in testis clustered cells}

ScRNA-seq datasets were analysed using the Seurat(version 4.0.2) and Harmony package (version 1.0). Marker genes for cell clustering were obtained using the FindAllMarkers function, and the SingleR package (version 1.4.1) was automatically annotated in combination with manual querying of the Human Cell Landscape (HCL) database (http://bis.zju.edu.cn/HCL/) for cell clustering.

\section{Constructing Trajectories of Hub Genes in Single-Cell datasets}

Monocle3 (version 1.0.0) is an R package dedicated to the analysis of scRNA-seq data, providing a variety of functions such as clustering, pseudotime, differential analysis and more. The developmental trajectory of sperm cells and Hub genes is constructed by using functions such as reduce_dimension, learn_graph and plot_genes_in_pseudotime.

\section{Results}

\section{Identification of DEGs in the transcriptome dataset}

7844 DEGs were obtained in the GSE103905 dataset, containing 4606 down-regulated genes and 3238 up-regulated genes (Fig.1A), while 4824 DEGs were obtained in the GSE103613 dataset, containing 2966 
down-regulated genes and 1858 up-regulated genes (Fig.1B). The Venn diagram showed 700 DEGs overlapping between the two datasets(Fig.1C).

\section{GO and KEGG enrichment analysis}

DAVID(https://david.ncifcrf.gov/)online analysis of GO functional enrichment and KEGG signalling pathway analysis of DEGs. In the BP category, significant enrichment was found in stabilization of membrane potential, memory and methylation (S.1A). In the MF category, significant enrichment was found in potassium ion leak channel activity, ATPase activity (S.1B). In the CC category, the mitochondrion, extracellular matrix and mitochondrial intermembrane space were significantly enriched (S.1C). In the KEGG enrichment analysis, it was found that the signalling pathways that may affect spermatogenesis are: metabolic pathways, huntington's disease and nicotinamide metabolism (S.1D).

\section{Construction of PPI networks and identification of Hub genes}

The testicular tissue-specific PPI networks of DEGs were analysed through the NetworkAnalyst website, and then, these networks were imported into Cytoscape to form a PPI of 254 nodes and 340 edges (Fig.2A). We used the Degree, DMNC, MCC and MNC algorithms in the cytoHubba plugin to obtain the Top 10 key genes for each algorithm (Fig.2 B-E). Finally, 4 genes (KIF2C,MRPS2,RPS15,TSFM) were repeated in more than 3 algorithms and they were considered as hub genes (Fig.2 F).

\section{Expression of Hub genes in sperm cells}

To further investigate the expression of the four Hub genes in sperm cells, we integrated scRNA-seq data from testicular tissues of normal human and KS patients and constructed a single-cell atlas formed by clustering 20 cells (Fig. 3 A). A total of 12 sperm cells were identified by similarity heatmap of cell clustering and marker genes (Fig. 3 B-C). They were: Leydig cell (LC), Myoid cell (MC), Macrophage, Sertoli cell (SC), Endothelial cell (EC) Spermatogonial stem cell (SSC), Early primary spermatocyte (EPS), Differentiating spermatogonia (DS), Late primary spermatocyte (LPS), Round spermatid(RS), Elongated spermatid(ES) and sperm (Fig. 3 D). Only KIF2C and RPS15 were expressed in the cell clusters, with RPS15 being expressed in all cells, whereas KIF2C gene expression was cell-specific and only expressed in the DS, ES, RS, EPS and sperm (Fig. 3 E-F).

\section{Differential expression of Hub genes in KS and normal groups}

In the single-cell dataset, where sperm cells were rare in KS patients, testicular somatic cells (SC,MC,EC,LC) and immune cells were predominant, whereas normal human testicular cells contained 12 species of testicular cells covering the whole process of spermatogenesis (S.2 A,B). The analysis showed that the RPS15 gene in KS was expressed at higher levels in all testicular cells than in normal subjects, whereas the expression level of the KIF2C gene was only expressed in the normal group and almost absent in the KS group (S.2 C,D).

\section{Pseudotime analysis of KIF2C and RPS15 in spermatocytes}


We verified that KIF2C and RPS15 were differentially expressed in sperm cells from the KS and the normal group, and it was necessary to accurately capture the developmental trajectories of these two genes in sperm cells. The developmental trajectory of sperm cells was constructed using monocle 3 software, and the results showed that a total of 11 branches were present in the sperm cells, and the branches were mainly distributed throughout the development from ES to sperm and in SC cells (Fig.4 A). During spermatogenesis, we successfully identified the initiating cell as SSC and the terminal cell as sperm (Fig. 4 B). We constructed trajectories of KIF2C and RPS15 in sperm development, and the results showed that RPS15 showed a downward trend from SSC to sperm formation. The expression of KIF2C showed an upward trend from SSC to sperm formation, indicating that KIF2C plays a crucial role in sperm development (Fig.4 C,D).

\section{Discussion}

$\mathrm{KS}$ is an important genetic factor in non-obstructive azoospermia and oligospermia (NOA), which is accompanied by impaired spermatogenesis, with $90 \%$ of patients presenting with azoospermia and $10 \%$ with severe oligospermia[15, 16]. However, the effect of the extra $X$ chromosome on germ cells remains a mystery, and the process of KS leading to spermatogenesis disorders is a complex process involving multiple regulatory mechanisms, of which the study of gene regulation at the transcriptional level has become a hot topic in recent years and has helped to elucidate the molecular mechanisms underlying KS spermatogenesis disorders.

Several studies have shown that alterations in transcriptome genes affect the development of KS spermatocytes with different clinical phenotypes[17].The transcriptome data from the current study showed that in the GSE103905 dataset, there were 7844 DEGs with 4606 down-regulated genes and 3238 up-regulated genes. In contrast, in the GSE103613 dataset, there were 4824 DEGs, 2966 down-regulated genes and 1858 up-regulated genes, with 700 DEGs overlapping between the two datasets. Subsequently, the GO functional annotated BP classification of DEGs resulted mainly in stabilization of membrane potential, memory and methylation. some of these studies have confirmed that KS is associated with predominantly genome-wide hypermethylation and less hypomethylated genomic regions[18, 19].The MF classification results are mainly in potassium ion leak channel activity and ATPase activity. The CC classification results are mainly in mitochondrion, extracellular matrix and mitochondrial intermembrane space $\llbracket K S$ patients with motor dysfunction and cognitive-psychological impairment are more likely to suffer from depression, anxiety, schizophrenia, autism, attention deficit disorder, and $20-50 \%$ of patients have intentional tremor[20,21].In addition to this, KS patients have a range of complications and are more likely to develop metabolic related diseases such as type 2 diabetes, dyslipidaemia, fatty liver, peripheral vascular disease, thromboembolic disease and endocrine related diseases[22]. These proven clinical signs and their complications are highly consistent with our results.

A testicular tissue-specific PPI network was constructed to obtain the top 10 key genes from four algorithms, Degree, DMNC, MCC and MNC, respectively, yielding a total of 40 genes. For the first time, 
KIF2C, MRPS2, RPS15 and TSFM were screened for possible close association with spermatogenic disorders in KS patients.

To investigate in depth the expression of these four genes in testicular cells, we obtained 20 clustered cells by integrating testicular cells from scRNA-seq, subsequently, a total of 12 spermatocytes were identified[23].Our results showed that only KIF2C and RPS15 were expressed in cell clusters, with RPS15 being expressed in all cell clusters, while KIF2C was expressed only in sperm cells, with cell specificity. Further differentiation by different populations showed that the RPS15 in KS was expressed at higher levels in all testicular cells than in normal individuals, whereas the KIF2C was expressed at levels only in the normal group and almost absent in the KS group. The above studies suggest that the expression of these two hub genes differs in the process of sperm development.

Clear developmental trajectories exist from spermatogonial stem cells to mature sperm cells. Using the monocle3, we successfully constructed developmental trajectories of sperm cells from single-cell datasets of normal and KS patients.SSC was correctly identified as the starting point of spermatogenesis, with multiplebranch occurring mainly during the development of ES to Sperm. Constructing trajectories of RPS15 and KIF2C in sperm development, RPS15 showed a downward trend from SSC cells to sperm, while KIF2C showed a small drop back from the beginning of DS expression to the peak in sperm cells. Our study suggests that there are differences in the roles played by these two Hub genes in sperm development. The RPS15 encodes a protein belonging to the S19P family of ribosomal proteins, which is a component of the $40 \mathrm{~S}$ subunit[24].As is typical for genes encoding ribosomal proteins, there are multiple processed pseudogenes of this gene dispersed through the genome. The RPS15 has been found to be activated in a variety of tumours, such as leukaemia, chronic lymphocytic leukaemia and prostate cancer, but no studies relating RPS15 to spermatogenesis have been seen[25, 26]. The KIF2C encodes a kinesin-like protein that functions as a microtubule-dependent molecular motor. The encoded protein depolymerises microtubules at the positive end, thereby facilitating mitotic chromosome segregation[27].Animal experiments have shown that KIF2C plays an important role in mitosis and meiosis in sperm cells[28], and mutations in KIF2C may cause sperm cells to become impaired during division and produce aneuploid gametes[29]. We therefore suggest that KIF2C plays an important role in the process of germ cell differentiation.

\section{Conclusion}

In summary, in this study, 700 DEGs and their GO and KEGG enrichment pathways were identified in KS and normal populations, and four Hub genes were screened. Finally, the developmental trajectories of sperm cells were constructed to identify that RPS15 and KIF2C genes may play an important role in sperm development. However, the mechanisms by which they affect sperm development may differ significantly and warrant further in-depth studies. These new findings provide important insights into the infertility of KS, and the two hub genes may be biomarkers for spermatogenic disorders, providing a strong basis for accurate diagnosis and precise treatment of KS. 


\section{Declarations}

\section{Ethics and consent to Publication}

The data for this study were obtained from a free public database and were exempted by the Ethics Committee of the Shenzhen Hospital of Southern Medical University. The research was conducted in accordance with the Declaration of Helsinki (revised 2013). All participants have given their consent to the publication of this thesis.

\section{Consent to Participate}

Written Informed consent was obtained from all participants.All authors have reached adulthood.

\section{Availability of Data and materials}

The datasets generated and analysed during the current study are available in the [GEO:GSE103905 and GSE103613] repository, [http://www.ncbi.nlm.nih.gov/geo/]".

\section{Funding}

The study is supported by Shenzhen Hospital of Southern Medical University, Research Promotion Funds for the Key Discipline Construction Program (NO. ZDXKKYTS006). The study received grants fromthe WU JIEPING MEDICAL FOUNDATION(NO.320.6750.2021-06-30) and the National Natural Science Foundation of China(NO.82002974).

\section{Competing Interest}

The authors declare that they have no conflict of interest.

\section{Authors contribution}

(I) Conception and design:H He,Y Zhou; (II) Administrative support: None; (III) Provision of study materials or patients: F Yu, T Huang; (IV) Collection and assembly of data:All authors; (V) Data analysis and interpretation: K Yan; (VI) Manuscript writing: All authors; (VII) Final approval of manuscript: All authors.

\section{Acknowledgement}

We acknowledge the GEO database for providing free transcriptome and single-cell sequencing data and thank the researchers who provided free data for this study.

\section{Author details}

${ }^{1}$ Clinical Laboratory Medicine Centre, Shenzhen Hospital, Southern Medical University, Shenzhen, 518108, China. 


\section{References}

1. Bojesen A and C H Gravholt. Klinefelter syndrome in clinical practice. Nat Clin Pract Urol. 2007; 4(4): 192-204.

2. Bojesen $A, S$ Juul, and $\mathrm{CH}$ Gravholt. Prenatal and postnatal prevalence of Klinefelter syndrome: a national registry study. J Clin Endocrinol Metab. 2003; 88(2): 622-6.

3. He H, Zhou H, Chen Y, Wangyou Y, and X Y. Chromosome and azoospermia factor in 626 males with severely impaired spermatogenesis. Journal of Chongqing Medical University 2014; 39(11): 1564-8.

4. Zitzmann $\mathrm{M}$ and $\mathrm{J}$ Rohayem. Gonadal dysfunction and beyond: Clinical challenges in children, adolescents, and adults with 47,XXY Klinefelter syndrome. Am J Med Genet C Semin Med Genet. 2020; 184(2): 302-312.

5. Bonomi M, V Rochira, D Pasquali, G Balercia, E A Jannini, A Ferlin, et al. Klinefelter syndrome (KS): genetics, clinical phenotype and hypogonadism. J Endocrinol Invest. 2017; 40(2): 123-134.

6. Zitzmann M, R Bongers, S Werler, N Bogdanova, J Wistuba, S Kliesch, et al. Gene expression patterns in relation to the clinical phenotype in Klinefelter syndrome. J Clin Endocrinol Metab. 2015; 100(3): E518-23.

7. Zganjar A, A Nangia, R Sokol, A Ryabets, and M K Samplaski. Fertility in Adolescents With Klinefelter Syndrome: A Survey of Current Clinical Practice. J Clin Endocrinol Metab. 2020; 105(4).

8. Bhasin S and R D Oates. Fertility Considerations in Adolescent Klinefelter Syndrome: Current Practice Patterns. J Clin Endocrinol Metab. 2020; 105(4).

9. Fainberg J, R P Hayden, and P N Schlegel. Fertility management of Klinefelter syndrome. Expert Rev Endocrinol Metab. 2019; 14(6): 369-380.

10. De Souza E, J K Morris, and E W Group. Case-control analysis of paternal age and trisomic anomalies. Arch Dis Child. 2010; 95(11): 893-7.

11. Deebel N A, A W Bradshaw, and H Sadri-Ardekani. Infertility considerations in klinefelter syndrome: From origin to management. Best Pract Res Clin Endocrinol Metab. 2020; 34(6): 101480.

12. Skakkebaek A, M Viuff, M M Nielsen, and C H Gravholt. Epigenetics and genomics in Klinefelter syndrome. Am J Med Genet C Semin Med Genet. 2020; 184(2): 216-225.

13. Gravholt C H, S Chang, M Wallentin, J Fedder, P Moore, and A Skakkebaek. Klinefelter Syndrome: Integrating Genetics, Neuropsychology, and Endocrinology. Endocr Rev. 2018; 39(4): 389-423.

14. Zhou G, O Soufan, J Ewald, R E W Hancock, N Basu, and J Xia. NetworkAnalyst 3.0: a visual analytics platform for comprehensive gene expression profiling and meta-analysis. Nucleic Acids Res. 2019; 47(W1): W234-W241.

15. Chang S, C F Christiansen, A Bojesen, S Juul, A B Munster, and C H Gravholt. Klinefelter syndrome and testosterone treatment: a national cohort study on thrombosis risk. Endocr Connect. 2020; 9(1): 34-43.

16. He H, F Yu, W Shen, K Chen, L Zhang, S Lou, et al. The Novel Key Genes of Non-obstructive Azoospermia Affect Spermatogenesis: Transcriptomic Analysis Based on RNA-Seq and scRNA-Seq 
Data. Front Genet. 2021; 12: 608629.

17. Belling K, F Russo, A B Jensen, M D Dalgaard, D Westergaard, E Rajpert-De Meyts, et al. Klinefelter syndrome comorbidities linked to increased $X$ chromosome gene dosage and altered protein interactome activity. Hum Mol Genet. 2017; 26(7): 1219-1229.

18. Wan E S, W Qiu, J Morrow, T H Beaty, J Hetmanski, B J Make, et al. Genome-wide site-specific differential methylation in the blood of individuals with Klinefelter syndrome. Mol Reprod Dev. 2015; 82(5): 377-86.

19. Zhang X, D Hong, S Ma, T Ward, M Ho, R Pattni, et al. Integrated functional genomic analyses of Klinefelter and Turner syndromes reveal global network effects of altered $\mathrm{X}$ chromosome dosage. Proc Natl Acad Sci U S A. 2020; 117(9): 4864-4873.

20. van Rijn S, A Aleman, H Swaab, T Krijn, G Vingerhoets, and R Kahn. What it is said versus how it is said: comprehension of affective prosody in men with Klinefelter $(47, \mathrm{XXY})$ syndrome. J Int Neuropsychol Soc. 2007; 13(6): 1065-70.

21. Ross J L, D P Roeltgen, G Stefanatos, R Benecke, M P Zeger, H Kushner, et al. Cognitive and motor development during childhood in boys with Klinefelter syndrome. Am J Med Genet A. 2008; 146A(6): 708-19.

22. Salzano A, M Arcopinto, A M Marra, E Bobbio, D Esposito, G Accardo, et al. Klinefelter syndrome, cardiovascular system, and thromboembolic disease: review of literature and clinical perspectives. Eur J Endocrinol. 2016; 175(1): R27-40.

23. Han X, Z Zhou, L Fei, H Sun, and G Guo. Construction of a human cell landscape at single-cell level. Nature. 2020; 581(7808).

24. Ntoufa S, M Gerousi, S Laidou, F Psomopoulos, G Tsiolas, T Moysiadis, et al. RPS15 mutations rewire RNA translation in chronic lymphocytic leukemia. Blood Adv. 2021; 5(13): 2788-2792.

25. Ljungstrom V, D Cortese, E Young, T Pandzic, L Mansouri, K Plevova, et al. Whole-exome sequencing in relapsing chronic lymphocytic leukemia: clinical impact of recurrent RPS15 mutations. Blood. 2016; 127(8): 1007-16.

26. Nikas J B and E G Nikas. Genome-Wide DNA Methylation Model for the Diagnosis of Prostate Cancer. ACS Omega. 2019; 4(12): 14895-14901.

27. Yuan, Juping, Ritter, Andreas, Louwen, Frank, et al. Molecular insight into the regulation and function of MCAK. Critical Reviews in Biochemistry \& Molecular Biology. 2016.

28. Parra M T, R Gomez, A Viera, E Llano, A M Pendas, J S Rufas, et al. Sequential assembly of centromeric proteins in male mouse meiosis. PLoS Genet. 2009; 5(3): e1000417.

29. Ems-McClung S C, K M Hertzer, X Zhang, M W Miller, and C E Walczak. The interplay of the N- and Cterminal domains of MCAK control microtubule depolymerization activity and spindle assembly. Mol Biol Cell. 2007; 18(1): 282-94.

\section{Figures}


a

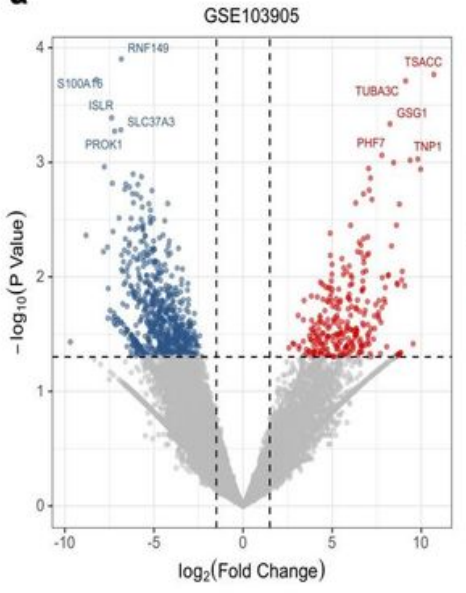

b

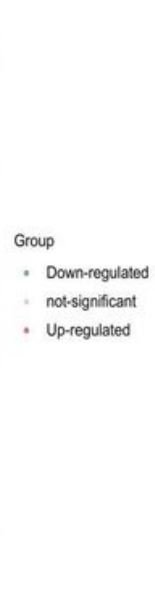

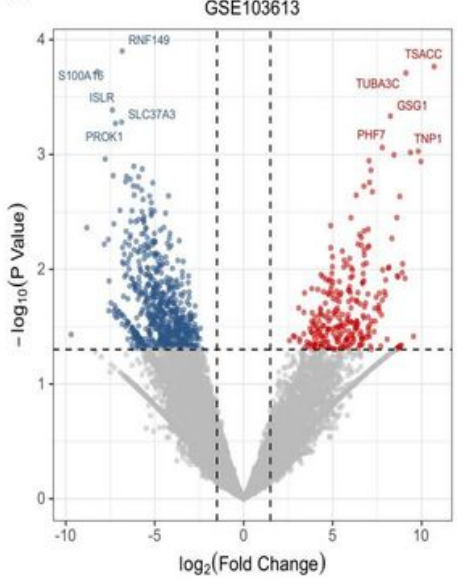

C

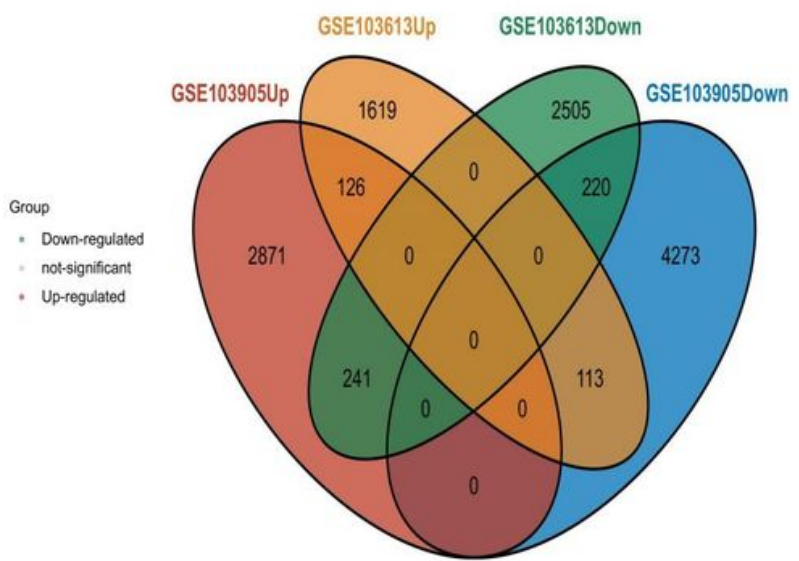

\section{Figure 1}

Identification of DEGs in KS and normal individuals, blue represents down-regulated genes and red represents up-regulated genes. (A) Volcano plot showing DEGs in the GSE103905 dataset.(B) Volcano plot showing DEGs in the GSE103613 dataset.(C) Wayne plot showing DEGs in the two datasets overlapping.

a

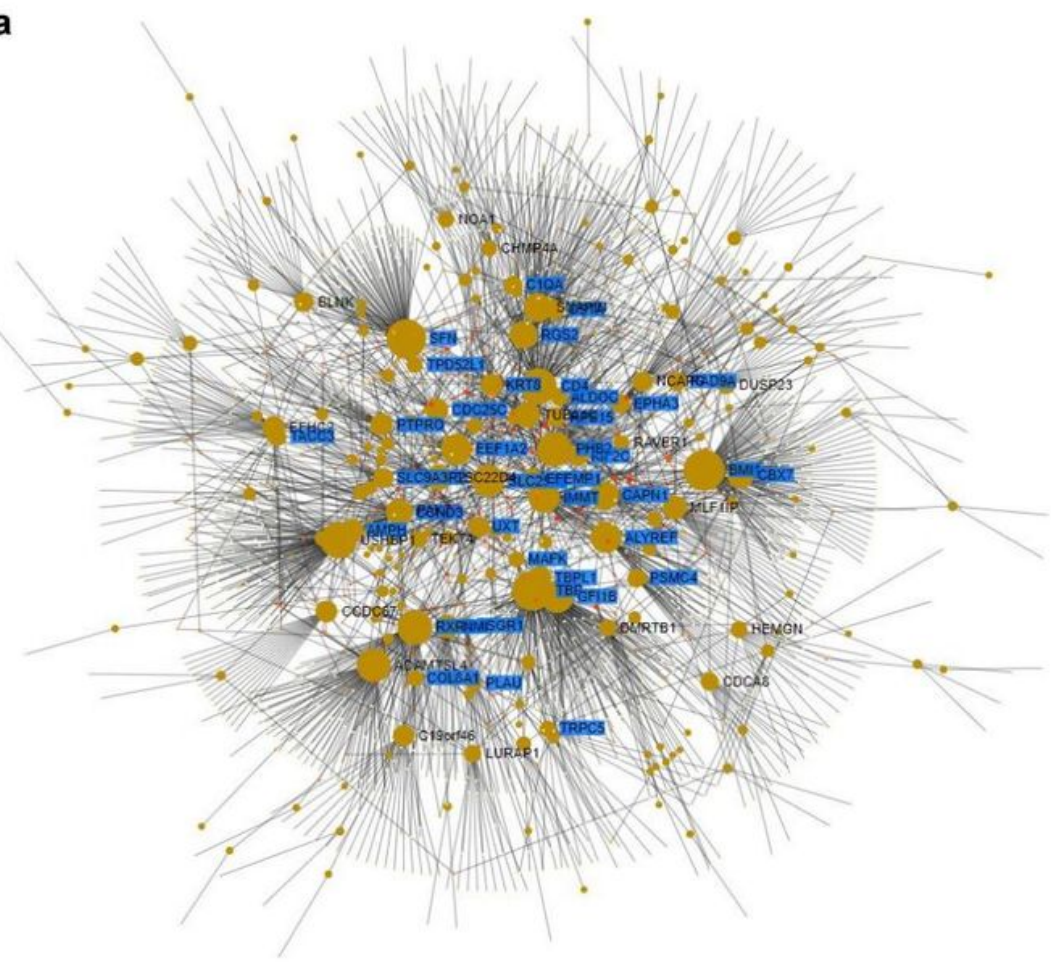

b

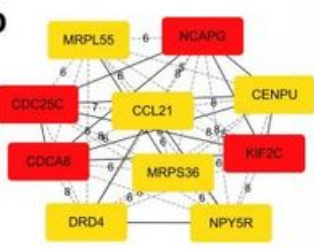

d

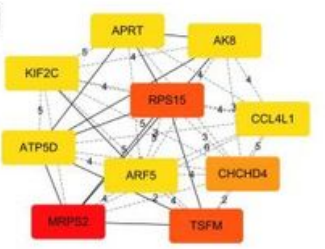

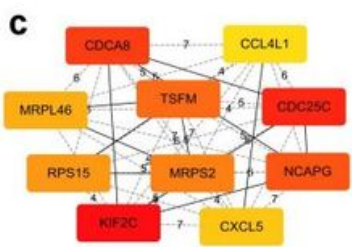

e

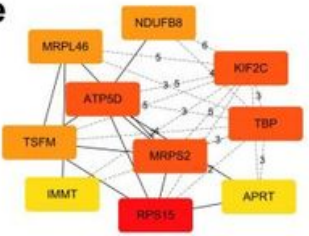

f

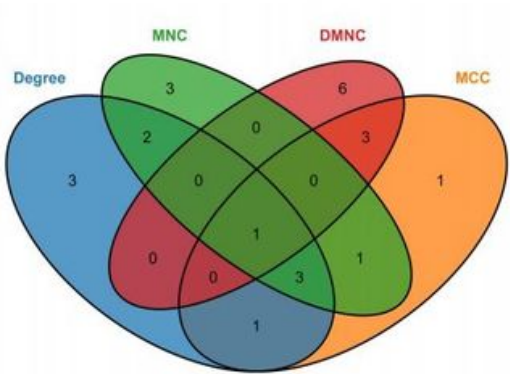

Figure 2 
Testicular tissue-specific PPI network and identification of Hub genes. (A) Testicular tissue-specific PPI network of differentially expressed genes. (B) TOP 10 key genes of the Degree algorithm.(C) TOP 10 key genes of the MCC algorithm.(D) TOP 10 hub genes of the MNC algorithm.(E) TOP 10 key genes of the DMNC algorithm.(F) Wayne diagram visualization of 4 Hub genes.
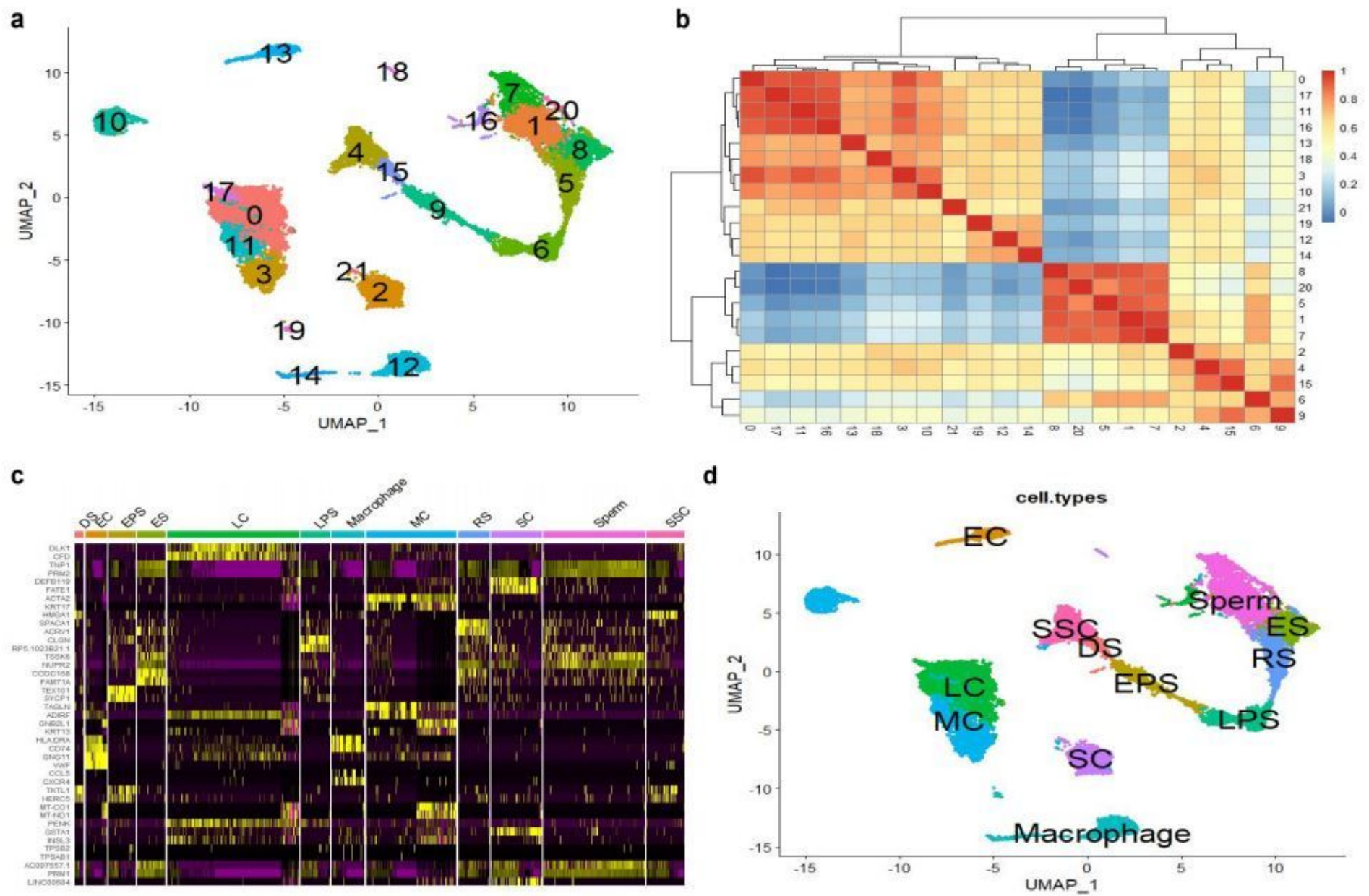

d
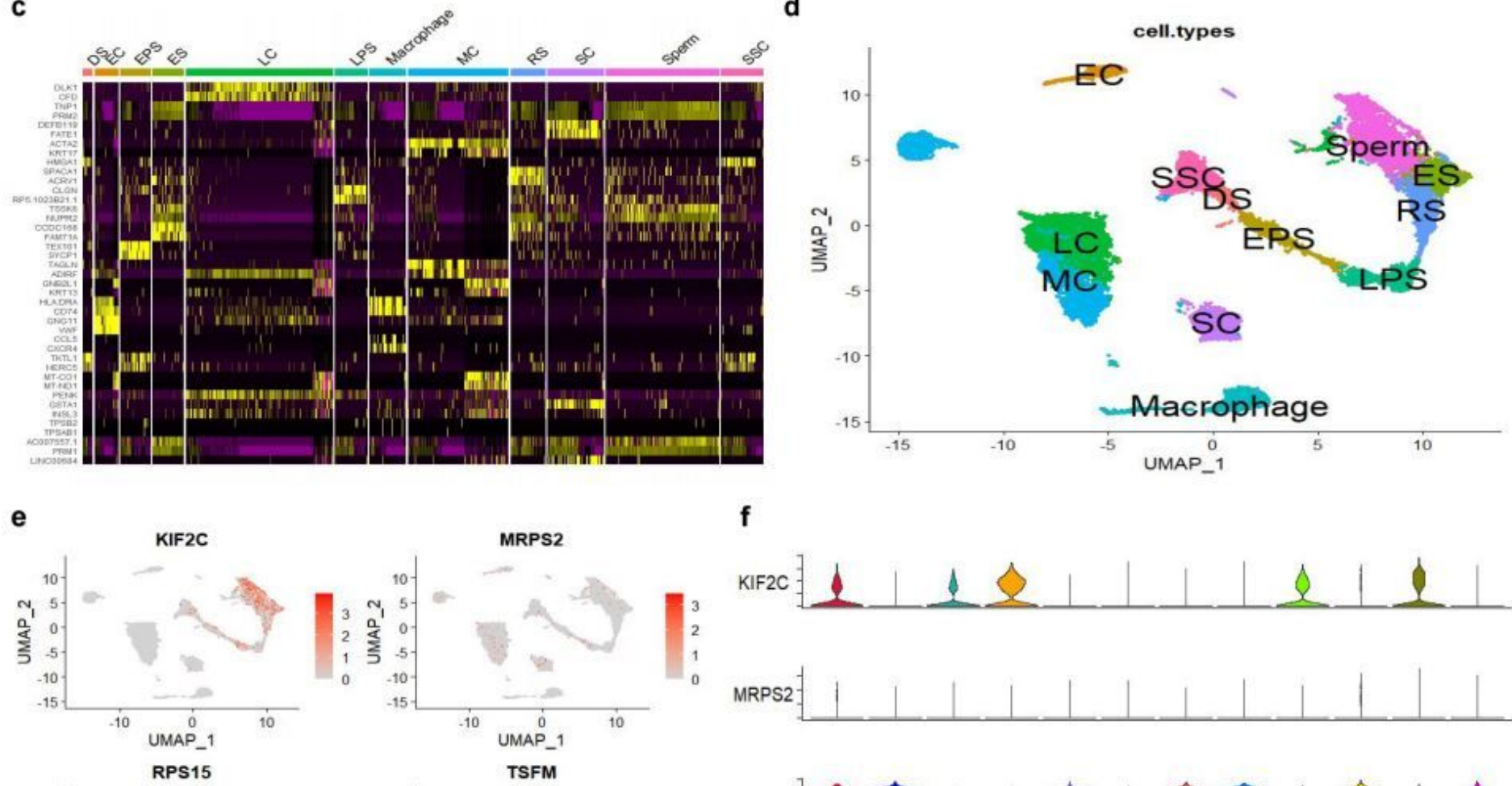

f
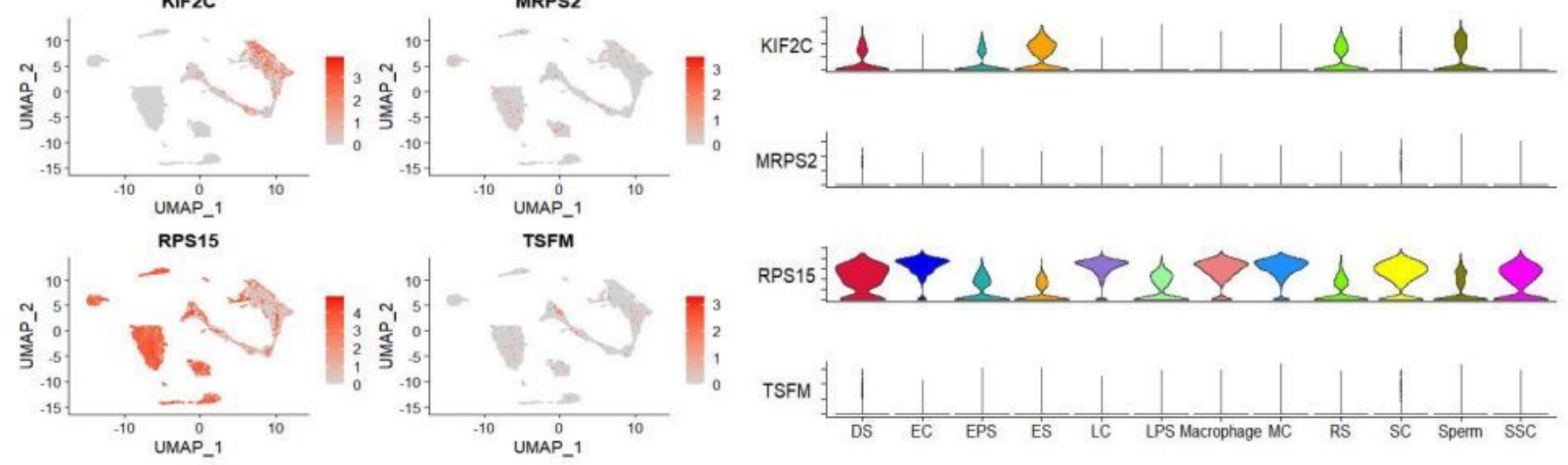

Figure 3 
Expression of Hub genes in testicular cells. (A) Integration of two single-cell sequencing datasets and UMAP visualization of 20 clustered cells. (B) Heatmap of correlation between cell clusters. (C) Heat map presenting Marker genes for cell clustering. (D) UMAP plots of 12 testicular cells. (E) Distribution map visualizing the expression of four hub genes in testicular cells. (F) Violin plot showing the expression of hub genes in 12 testicular cells.
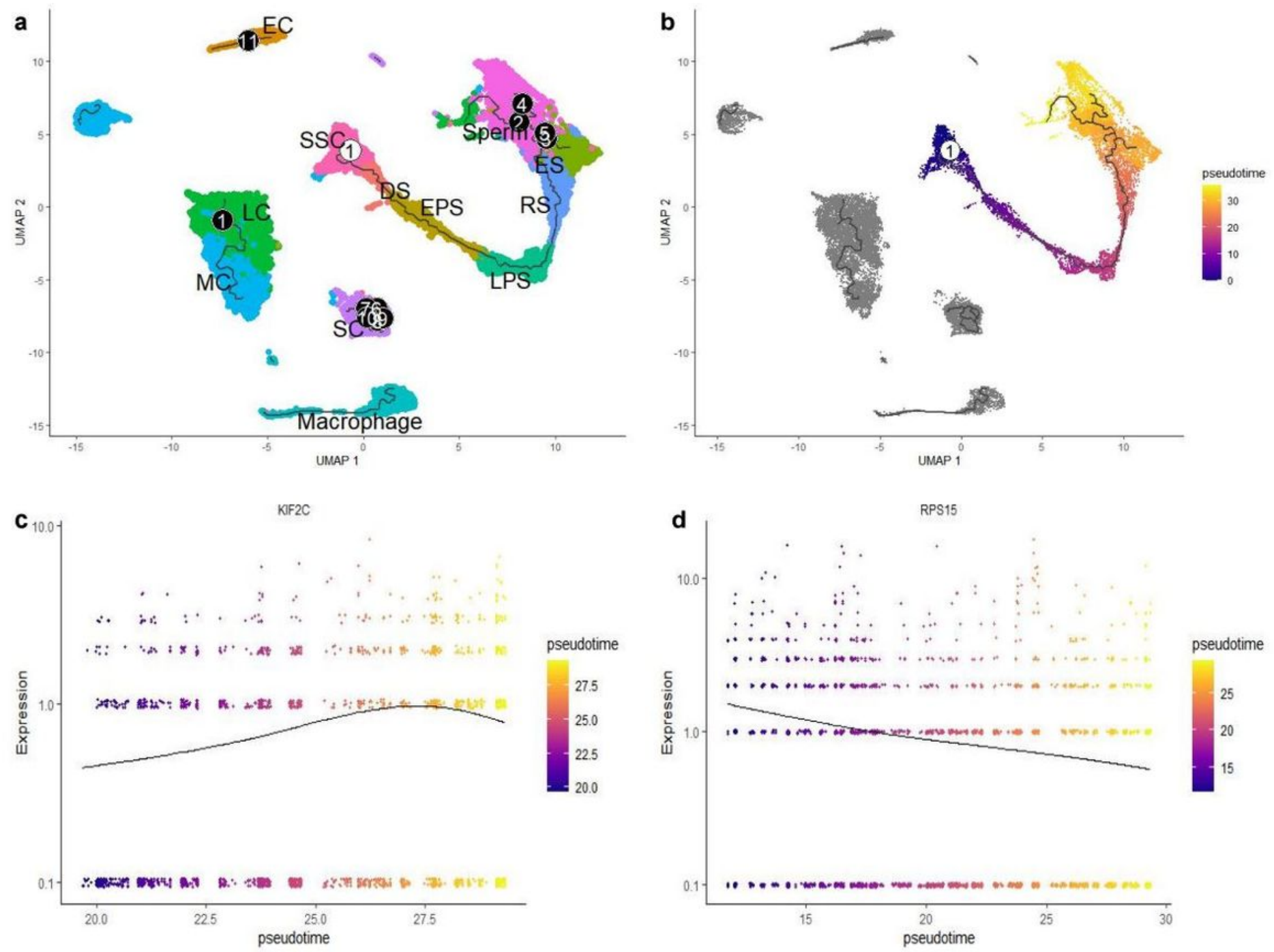

\section{Figure 4}

Pseudotime analysis of testicular cell development.(A) Developmental trajectory of testicular cells, numbers in white on a black background indicate the branch that occurs in the developmental trajectory

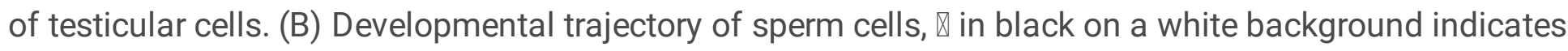
that sperm development begins at the SSC. (C) Analysis of the trajectory of KIF2C throughout the development of sperm cells. (D) Analysis of the trajectory of RPS15 in the full development of sperm cells.

\section{Supplementary Files}


This is a list of supplementary files associated with this preprint. Click to download.

- Supplemental1.pdf

- Supplemental2.pdf 